\title{
Removal of Integrated Hepatitis B Virus DNA Using CRISPR-Cas9
}

\begin{abstract}
Hao $\mathrm{Li}^{1+}$, Chunyu Sheng ${ }^{1+}$, Shan Wang ${ }^{1+}$, Lang Yang ${ }^{1}$, Yuan Liang ${ }^{1}$, Yong Huang ${ }^{2}$, Hongbo Liu ${ }^{1}$, Peng Li ${ }^{1}$, Chaojie Yang ${ }^{1}$, Xiaoxia Yang ${ }^{1}$, Leili Jia ${ }^{1}$, Jing Xie ${ }^{1}$, Ligui Wang ${ }^{1}$, Rongzhang Hao ${ }^{1}$, Xinying $\mathrm{Du}^{1}$, Dongping $\mathrm{Xu}^{3}$, Jianjun Zhou ${ }^{4,5}$, Mingzhen $\mathrm{Li}^{6}$, Yansong Sun ${ }^{1}$, Yigang Tong ${ }^{2}$, Qiao $\mathrm{Li}^{7}$, Shaofu Qiu ${ }^{1 *}$ and Hongbin Song ${ }^{1 *}$

${ }^{1}$ Center for Infectious Disease Control, Institute of Disease Control and Prevention, Academy of Military Medical Sciences, Beijing, China, ${ }^{2}$ State Key Laboratory of Pathogen and Biosecurity, Beijing Institute of Microbiology and Epidemiology, Beijing, China, ${ }^{3}$ Research Centre for Liver Failure, Beijing 302nd Hospital, Beijing, China, ${ }^{4}$ Research Center for Translational Medicine, Cancer Stem Cell Institute, East Hospital, Tongji University School of Medicine, Shanghai, China, ${ }^{5}$ Gladcan Consulting Company, Beijing, China, ${ }^{6}$ Research and Development Department, Beijing Center for Physical and Chemical Analysis, Beijing, China, ' Department of Surgery, University of Michigan, Ann Arbor, MI, USA
\end{abstract}

The presence of hepatitis B virus (HBV) covalently closed circular DNA (cccDNA) and the permanent integration of HBV DNA into the host genome confers the risk of viral

OPEN ACCESS

Edited by:

Slobodan Paessler,

University of Texas Medical Branch,

USA

Reviewed by:

Qiao Fei,

Medical University of South Carolina,

USA

ShiXing Tang,

Southern Medical University, China

Guangping Tai,

University of Edinburgh, UK

*Correspondence:

Hongbin Song

hongbinsong@263.net

Shaofu Qiu

qiushf0613@hotmail.com

${ }^{\dagger}$ These authors have contributed equally to this work.

Received: 23 January 2017 Accepted: 07 March 2017 Published: 22 March 2017

Citation:

$L i H$, Sheng $C$, Wang S, Yang L, Liang Y, Huang Y, Liu H, Li P, Yang C, Yang $X$, Jia L, Xie J, Wang L, Hao R,

DuX, Xu D, Zhou J, Li M, Sun Y, Tong Y, Li Q, Qiu S and Song H (2017)

Removal of Integrated Hepatitis $B$ Virus DNA Using CRISPR-Cas9.

Front. Cell. Infect. Microbiol. 7:91.

doi: 10.3389/fcimb.2017.00091 reactivation and hepatocellular carcinoma. Nucleoside/nucleotide analogs alone have little or no capacity to eliminate replicative HBV templates consisting of cccDNA or integrated HBV DNA. Recently, CRISPR/Cas9 technology has been widely applied as a promising genome-editing tool, and HBV-specific CRISPR-Cas9 systems were shown to effectively mediate HBV cccDNA disruption. However, the integrated HBV DNA fragments are considered as important pro-oncogenic properties and it serves as an important template for viral replication and expression in stable HBV cell line. In this study, we completely excised a full-length 3,175-bp integrated HBV DNA fragment and disrupted HBV cccDNA in a stable HBV cell line. In HBV-excised cell line, the HBV cccDNA inside cells, supernatant HBV DNA, HBsAg, and HBeAg remained below the negative critical values for more than 10 months. Besides, by whole genome sequencing, we analyzed off-target effects and excluded cell contamination. It is the first time that the HBV infection has been fully eradicated in a stable HBV cell line. These findings demonstrate that the CRISPR-Cas9 system is a potentially powerful tool capable of promoting a radical or "sterile" HBV cure.

Keywords: hepatitis B virus, CRISPR-Cas9, integrated HBV DNA, HBV cccDNA, whole genome sequencing

\section{INTRODUCTION}

Chronic hepatitis $\mathrm{B}(\mathrm{CHB})$ is a major public health problem worldwide, with $350-400$ million chronic HBV (hepatitis B virus) carriers (Seo and Yano, 2014). HBV infection results in 0.5-1 million deaths per year due to cirrhosis of the liver, hepatocellular carcinoma, and liver failure (Kao and Chen, 2002; Roberts and Gores, 2005). HBV covalently closed circular DNA (cccDNA) is

Abbreviations: HBV, hepatitis B virus; NAs, nucleoside/nucleotide analogs; cccDNA, covalently closed circular DNA; CRISPR, clustered regularly interspaced short palindromic repeat; gRNA, guide RNA; PAM, protospacer adjacent motif; DSBs, double-strand breaks; HBS, hepatitis B surface antigen; indel, insertion or deletion; SD, standard deviation; NHEJ, Non-homologous end joining; PSAD, plasmid safe ATP-dependent DNase; RCA, rolling circle amplification. 
very stable in $\mathrm{CHB}$ patients and serves as the template for viral mRNA and pre-genomic RNA synthesis (Moraleda et al., 1997; Dandri et al., 2000; Gish et al., 2015; Guo and Guo, 2015; Nassal, 2015). The integration of HBV DNA into the host genome can cause alterations of the host genome, leading to changes in genes associated with cell proliferation, differentiation, and survival (Bonilla Guerrero and Roberts, 2005; Feitelson and Lee, 2007; Jiang S. et al., 2012; Jiang Z. et al., 2012; Sung et al., 2012; Xu et al., 2014). HBV DNA integration is also an important factor in hepatocarcinogenesis (Bréchot, 2004; Sung et al., 2012; Tarocchi et al., 2014; Xu et al., 2014). Current anti-HBV treatments with either nucleoside/nucleotide analogs (NAs) or interferon do not cure $\mathrm{CHB}$, and relapses are common (Bang and Kim, 2014). Although NAs can inhibit viral reverse transcriptase and suppress HBV replication, these drugs alone have little or no ability to eliminate replicative $\mathrm{HBV}$ templates comprising cccDNA (Moraleda et al., 1997; Dandri et al., 2000) or integrated HBV DNA (Zucman-Rossi and Laurent-Puig, 2007). Given the shortcomings of current therapeutic options, there is a need to approach $\mathrm{CHB}$ in a fundamentally different way (Shaw et al., 2006; Sharon and Chu, 2008).

A new genome-editing tool, CRISPR-Cas9, was recently developed based on the bacterial immune system's clustered regularly interspaced short palindromic repeats (CRISPRs) (Qi et al., 2013). Zinc Finger Nucleases (ZFNs) and Transcription activator-like effectors nucleases (TALENs) have been under investigation for their capacity to specifically disrupt HBV genomes in vitro and in vivo (Cradick et al., 2010; Bloom et al., 2013; Chen et al., 2014). However, compared with ZFNs and TALENs, the CRISPR/Cas9 system can be more easily reprogrammed and delivered both in vitro and in vivo to cleave virtually any DNA sequence by simply redesigning the guide RNAs (gRNAs), which is predicated to be a promising genome-editing tool with broad applications (Qi et al., 2013; Ran et al., 2013; Zhang et al., 2014). Using CRISPR-Cas9, Hu et al. completely excised the full length of integrated HIV proviral DNA in a stable HIV monoclonal cell line (Hu et al., 2014). In these studies, HBV-specific CRISPR-Cas9 systems effectively mediated gene disruption in HBV templates in expression vectors (Lin et al., 2014; Liu et al., 2015) and HBV cccDNA (Seeger and Sohn, 2014; Kennedy et al., 2015; Zhen et al., 2015a) both in vitro and in vivo. However, none of these studies demonstrated removal of the full-length integrated HBV DNA and subgenomic integrated HBV DNA fragments in a stable HBV cell line (Lin et al., 2014; Seeger and Sohn, 2014; Kennedy et al., 2015; Liu et al., 2015; Zhen et al., 2015a). These shortcomings largely limited the prospect of developing a fundamental therapeutic method of viral eradication through CRISPR-Cas9. Several studies have proposed that removal of integrated HBV DNA from the host genome is a necessary measure to recover the stability of the chromosome and cure HBV-related HCC (Peng et al., 2015; Ramanan et al., 2015; Wang et al., 2015). In a pioneering study, Karimova et al. disrupted integrated $\mathrm{HBV}$ DNA, using an integrated HBV reporter sequence in HeLa and HEK293 cell lines (Karimova et al., 2015). In HBV-infected cells, the existence of many different forms of episomal HBV DNA (Tuttleman et al., 1986) and multiple integrated HBV sites in different chromosomes (Matsubara and Tokino, 1990) made it cumbersome to specifically amplify the full length of integrated HBV DNA sites in a stable HBV cell line, using Alu-/LM-PCR. Also, the limitations of the short reads generated by next-generation sequencing (NGS) meant that HBV DNA integration sites could only be inferred from paired-end reads containing both human and viral sequences (Hai et al., 2014). In a previous study, we established a stable HBV cell line, HepG2.A64 (CCTCC C 201163), using genotype C HBV strains (GenBank: HQ638218.1) isolated from hepatitis B patients (Wei-ming et al., 2014). Compared with HepG2.2.15, this cell line could produce more antigens, virions, and $\mathrm{HBV} \operatorname{cccDNA}$, and was easier to cultivate and transfect. HBV transfected in HepG2.A64 cells contained entecavir-resistant mutations, which had already been used in drug resistance investigations (Liu et al., 2016). Moreover, we employed CRISPR-Cas9 to disrupt HBV cccDNA and inhibit viral replication in this cell line in our previous study (Li et al., 2016). In this study, not only HBV cccDNA, we also removed the full length of integrated HBV DNA, which means that we achieved a "sterile" eradication of HBV infection in this stable HBV cell line.

\section{MATERIALS AND METHODS}

\section{Plasmid Preparation}

The Cas9/gRNA dual-expression vector pSpCas9(BB)-2A-Puro (PX459) was a gift from Feng Zhang (Addgene plasmid \#48139) and was constructed according to a previously described protocol (Ran et al., 2013). Using a gRNA prediction tool (http://crispr. mit.edu/), five candidate target sequences were derived from the HBV genome (GenBank accession number HQ638218). These protospacers were subsequently inserted into dual-expression vectors under the control of the U6 promoter. Plasmids were purified using the EndoFree Plasmid Maxi Kit (Qiagen, Germany).

\section{Cell Culture and Transfections}

All cells were maintained in Dulbecco's modified Eagle medium supplemented with $10 \%$ fetal calf serum at $37^{\circ} \mathrm{C}$ and $5 \% \mathrm{CO}_{2}$. The HepG2.A64 (CCTCC C 201163) monoclonal cell line was established from HepG2 cells transfected with an HBV plasmid pTriexHBV1.1 containing 1.1 copies of HBV DNA (GenBank: HQ638218.1) (Wei-ming et al., 2014). This monoclonal cell line sustainably produced HBsAg, HBeAg, and HBV DNA. A total of $2 \times 10^{6}$ A64 cells were seeded onto $10-\mathrm{cm}$ plates 24 $\mathrm{h}$ before transfection. The Cas $9 / \mathrm{gRNA}$ co-expression vectors were transfected into A64 cells using Lipofectamine LTX (Life Technologies, US). Puromycin $(1 \mu \mathrm{g} / \mu \mathrm{l})$ was used to increase transfection efficiency (Figure S1). At $72 \mathrm{~h}$ after transfection, transfected cells were seeded onto 24 -well-plates $\left(\sim 4 \times 10^{4}\right.$ cells per well) for subsequent analysis. For subcloning, stable clones were subcultured after transfection and underwent puromycin selection by a limiting dilution method in 96-well-plates, and single cell-derived subclones were maintained for further studies. 


\section{T7El Assay}

The genomic region surrounding the target regions $\mathrm{A}$ and $\mathrm{B}$ was amplified by PCR; specific primers were designed with primer premier 5.0 (Table S1). All PCR products were verified by Sanger sequencing and subjected to a re-annealing process to induce heteroduplex formation. After re-annealing, products were treated with T7EI (New England Biolabs, US) at $37^{\circ} \mathrm{C}$ for 30 min and analyzed using 3\% agarose gels. Gels were imaged using a gel imaging system (Bio-Rad, US).

\section{Immunoassay and qPCR}

HBsAg and HBeAg were measured in the cell supernatants, using an ARCHITECT i2000 SR system (Abbott, US); data were reported in IU/ml for HBsAg and S/CO for HBeAg. Cell culture supernatants were extracted and tested for the presence of $\mathrm{HBV}$ DNA by real-time PCR. To quantitate HBV cccDNA, circular duplex DNA was subjected to PlasmidSafe ATP-dependent DNase (Epicentre, US), which has low activity toward HBV rcDNA, dsDNA, and ssDNA. Rolling circle amplification (RCA) was then performed to selectively amplify circular DNA. Based on the RCA products, HBV cccDNA was amplified and quantitated by TaqMan real-time PCR using specific cccDNA-selective primers and a probe targeting the gap region between DR1 and DR2 of the HBV genome (Zhong et al., 2011). To detect genomic DNA contamination, primer sets that amplify the HBV S DNA or control cellular genomic sequences located in the A1AT gene were used (Table S1). To quantitate the cell number, specific primers for human $\beta$-actin were used for real-time PCR.

\section{Whole-Genome Sequencing and Bioinformatics Analysis}

Genomic DNA was isolated by a cetyltrimethylammonium bromide (CTAB)-based extraction method. DNA samples and genomic DNA libraries were prepared by the NGS facility at the Biomarker Technologies Company. All libraries were sequenced with paired-end 150-bp reads in two Illumina Rapid Run flow cells, using a HiSeq X 10 instrument (Illumina). Demultiplexed read data from the sequenced libraries were sent to the Biomarker Technologies Company for bioinformatics analysis. Briefly, the raw reads were mapped against the human genome (hg19), using BWA. A genomic analysis toolkit (GATK, version 2.8.1) was used for removal of duplicate reads, local alignment, base quality recalibration, and indel calling.

\section{Statistical Analysis}

For statistical analysis, Student's $t$-test was performed using the SAS software suite. $P<0.05$ was considered significant. Error bars represent the SEM of at least three independent experiments.

\section{RESULTS}

\section{Analysis of Integrated HBV DNA and Rationale for Selection of the CRISPR-Cas9 Target Site}

We used the stable HBV cell line HepG2.A64 (CCTCC C 201163, hereafter referred to as "A64") as a cell model. The full length of integrated HBV DNA in this cell line was dependent on a foreign promoter (CMV chicken $\beta$-actin promoter) instead of viral promoters, which enabled amplification of the full-length replication-competent integrated HBV DNA, using a specific primer (P1) located in the foreign promoter region (Figure 1A). To ensure that the PCR products of the primers (P1 and P2) were the integrated HBV DNA rather than the fragment on pTriexHBV1.1, we used Plasmid-Safe ATP-Dependent DNase (PSAD) to extract the circular duplex DNA. HBV-specific primers (HBSF\&R) and genome-specific primers (A1ATF\&R) were used as the positive and negative controls, respectively, to evaluate the effect of the circular duplex DNA on extraction. The P1 primer and the HBV S gene-specific primer P3 did not amplify the circular duplex DNA (Figures 1C,D), indicating that there was no circular pTriexHBV1.1 in the stable HBV cell line A64 and that primer P1 was an integrated HBV DNAspecific primer. Next, we performed long-range PCR, using A64 genomic DNA with integrated HBV DNA-specific primers (P1 and P2) with a Phusion High-Fidelity PCR Kit (NEB, US), following the manufacturer's protocol. Sequencing of the PCR products revealed a 4,049-bp DNA fragment representing the 3,362-bp integrated HBV DNA (1.1 copies) plus a flanking 687bp pTriexHBV1.1-derived sequence (Figure 1B). The 3,362-bp integrated HBV DNA contained an entire 3,173-bp HBV genome and a 189-bp repeat sequence of the HBV core region. To remove the full-length integrated HBV DNA, we employed one gRNA targeting the two repeat regions of the integrated HBV DNA, which was expected to be more efficient in transfection and have lower off-target potential than the use of two gRNAs (Figure 1A). By online efficiency prediction (Hsu et al., 2013; Mali et al., 2013), we identified five gRNA targets with fewer off-target effects on the host genome (Table S2) and constructed the corresponding CRISPR-Cas9 systems.

\section{Specific Inactivation of HBV Replication by CRISPR-Cas9}

To assess the capacity to generate apparent cleavage at both ends of the integrated HBV DNA, we performed a mismatchsensitive T7 endonuclease I (T7EI) assay (Ran et al., 2013) after transfecting the five gRNAs into the A64 cell line (Figure 2A). All of the systems introduced double-strand breaks (DSBs) into both ends of the integrated HBV DNA, but gRNA-69 was the most efficient system (Figure 2A). Next, to determine the knockout efficiency of the gRNAs in A64 cells, we used an ARCHITECT HBsAg and HBeAg reagent kit to determine the amounts of HBeAg and HBsAg in the cell culture supernatants on 16 consecutive days post-transfection. At day 9 after transfection, compared with that in the gRNA-empty group, $\mathrm{HBeAg}$ concentrations were reduced $83.13 \pm 0.14 \%$ in the gRNA-91-treated group, $80.53 \pm 2.43 \%$ in the gRNA-69treated group, $70.71 \pm 2.09 \%$ in the gRNA-62-treated group, and $76.50 \pm 0.27 \%$ in the gRNA-60-treated group. HBsAg concentrations were reduced $87.38 \pm 1.56 \%$ in the gRNA-91treated group, $86.49 \pm 1.79 \%$ in the gRNA-69-treated group, $80.07 \pm 1.01 \%$ in the gRNA-62-treated group, and $82.55 \pm 0.78 \%$ in the gRNA-60-treated group. HBV DNA concentrations were reduced $91.72 \pm 1.55 \%$ in the gRNA-91-treated group, 
A



C

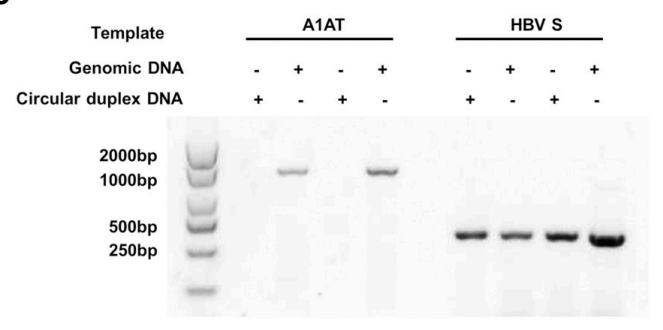

B

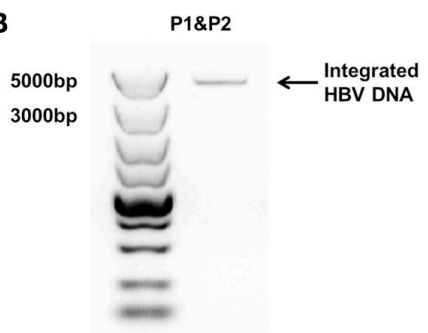

D

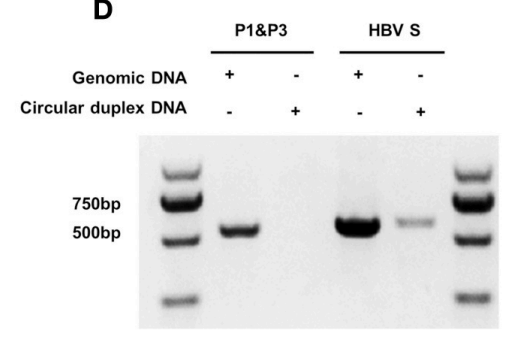

FIGURE 1 | Analysis of integrated HBV DNA in the stable HBV cell line A64. (A) Integrated HBV DNA in the stable HBV A64 cell line and the gRNA target sites in the repeat region of the $1.1 \mathrm{HBV}$ genome copy. (B) The 4,049-bp DNA fragment representing the 3,362-bp integrated HBV DNA (1.1 copies) plus a 687-bp pTriexHBV1.1-derived flanking sequence was efficiently amplified from cellular genomic DNA using the integrated HBV-specific primers P1 and P2. (C) PCR analysis using the A1AT and HBV S-gene primer sets conducted on total genomic DNA and circular duplex DNA to assess the effect of extraction on circular duplex DNA. (D) PCR analysis of the integrated HBV DNA or circular duplex DNA isolated from cells using the P1/P3 and S-gene primer sets. Using P1 and the HBV core region-specific primer P3, the HBV S-gene amplicons were predicted to be 542- and 572-bp, respectively. Primers P1/P3 did not amplify circular duplex DNA.

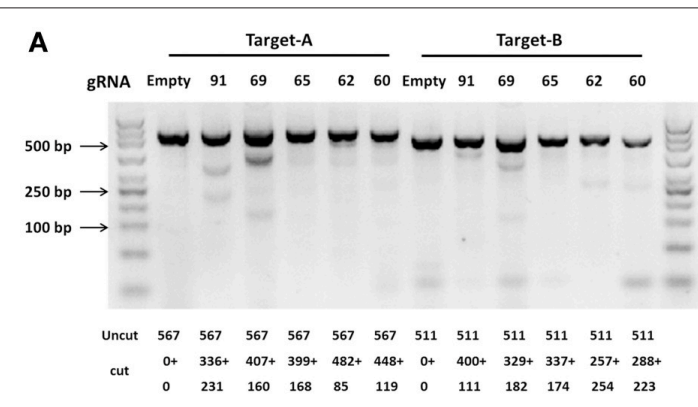

C



B



D



FIGURE 2 | Inhibition of both HBV antigen expression and HBV replication by CRISPR-Cas9 in A64 cells after transfection. (A) DNA extracted from A64 cells transfected with gRNA-91, gRNA-69, gRNA-65, gRNA-62, and gRNA-60 was analyzed by a T7El assay. Predicted sizes of uncut and cut bands are indicated. (B-D) Inhibition of HBsAg, HBV DNA, and HBeAg in cell culture supernatants at the indicated time points after transfection of A64 cells with gRNA-91, gRNA-69, gRNA-65, gRNA-62, and gRNA-60.

$89.21 \pm 2.72 \%$ in the gRNA-69-treated group, $80.30 \pm 1.30 \%$ in the gRNA-62-treated group, and $86.95 \pm 1.93 \%$ in the gRNA-60 treated-group. It is noteworthy that the suppression of HBsAg and HBV DNA was slightly higher than that of HBeAg. During
16 consecutive days after transfection, $\mathrm{HBV}$ DNA, HBeAg, and HBsAg concentrations in culture supernatants were low in the gRNA-91, gRNA-69, gRNA-62, and gRNA-60 groups (Figures 2B,D) compared with those in the gRNA-empty group 
(Figure 2C). Neither the HBV DNA nor HBV antigens have been reduced in the cells transfected with gRNA-65. Moreover, at 10 and 14 day post-transfection, the entire group showed a drop-off in suppression of HBeAg and HBV DNA. This phenomenon was expected due to the loss cells during medium changing.

\section{CRISPR-Cas9 Excised the Full-Length Integrated HBV Genome and Disrupted HBV cccDNA}

We expected that expression of the gRNAs in A64 cells would result in the deletion of the entire 3,173-bp HBV genome between the A and B target sites. By long-range PCR analysis, we found one subclone, HepG2.A64-69-7 (A64 transfected with gRNA-69, hereafter referred to as "69-7"), which contained a complete deletion of the entire 3,173-bp integrated HBV genome
(Figure 3A). An 873-bp fragment representing the predicted segment resulting from its flanking region was amplified (Figure 3A), suggesting gRNA-69 enabled Cas9 to excise the full-length integrated HBV genome segment. Sequence analysis demonstrated that the 873-bp fragment included 687-bp from the integrated pTriexHBV1.1-derived flanking sequence and a 186-bp HBV repeat core region sequence, with a 3-bp deletion at the gRNA-69 target site, which was expected to result from gRNA-69-guided cleavage and repair (Figure 3B).

Next, we performed quantitative analysis of HBsAg and HBeAg in the supernatant (Figures S1A,B) and real-time PCR analysis of supernatant HBV DNA and HBV cccDNA (Figures S1C,D). The HBV DNA in the supernatant from the gRNA-empty-treated group (subclone K-15) had a concentration of $775,033 \pm 29,868 \mathrm{IU} / \mathrm{ml}$ at day 10 after continuous cultivation, whereas the concentration of the HBV DNA in the supernatant
A

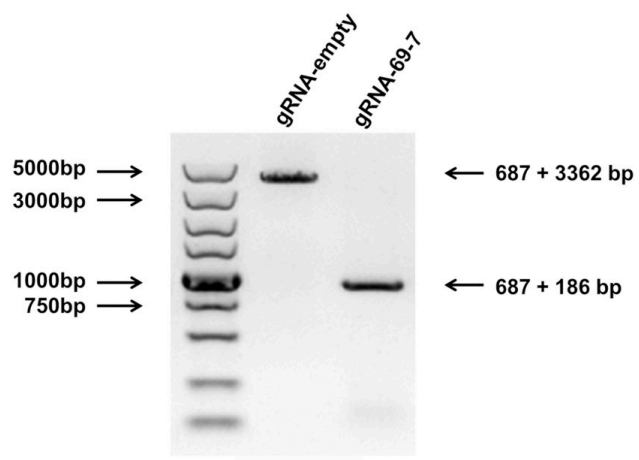

B



C

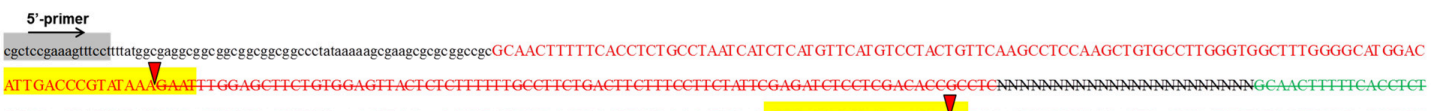

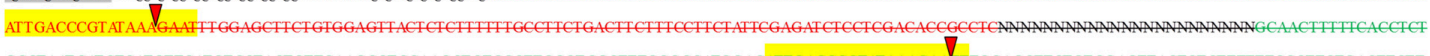
GCCTAATCATCTCATGTTCATGTCCTACTGTTCAAGCCTCCAAGCT GTGECTTGGGT GGCTTTGGGGCATGGACATTGACCCGTATAAAGANTTTGGAGCTTCT GT GGAGTT ACTCTCTTTTTTGCCTTCTGACTTCTT



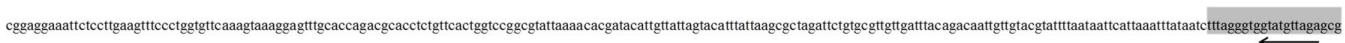

D

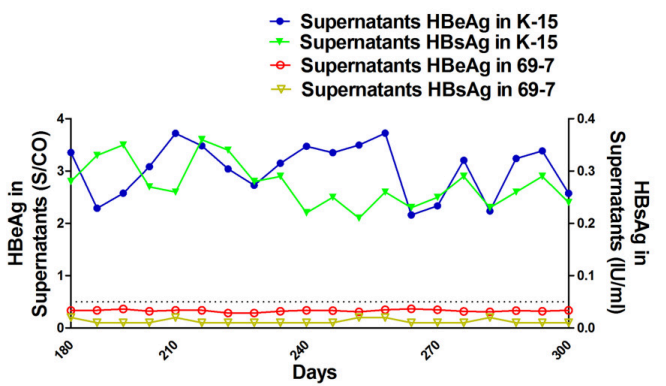

E

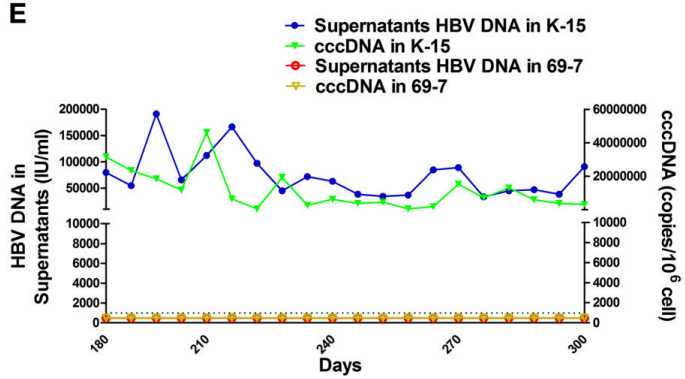

FIGURE 3 | CRISPR-Cas9/gRNA-69 efficiently removed the integrated HBV genome from a stable HBV cell line. (A) Analysis of PCR amplicon lengths using a primer pair (P1 and P2) targeting the integrated HBV-flanking sequence revealed elimination of the full-length integrated HBV genome (3,173-bp), leaving one fragment (873-bp predicted segment from its flanking region). (B) Diagram showing excision of the full-length integrated HBV genome. The remaining fragment included the expected 687-bp from the integrated HBV flanking sequence and a 186-bp HBV repeat core region sequence. (C) Sanger sequencing of the remaining fragment (873-bp) showing the HBV flanking sequence (small letters, 687-bp) and the partial sequences (189 - $3=186-\mathrm{bp})$ of the integrated HBV repeat region B (green) and repeat region A (red) with a 3-bp deletion around the gRNA-69 targeting site (yellow-highlighted). Elimination of the full-length integrated HBV genome is indicated by a strikethrough. (D,E) The amounts of HBeAg, HBsAg and HBV DNA in cell culture supernatants and HBV cccDNA in the gRNA-empty-treated group (K-15) and gRNA-69-treated group (69-7) over 300 consecutive days. The HBsAg and HBeAg test results in the gRNA-69-treated group (69-7) were always under the negative threshold $(0.05 \mathrm{IU} / \mathrm{ml}$ for HBsAg and $1 \mathrm{~S} / \mathrm{CO}$ for HBeAg), and the amounts of HBV DNA and HBV cccDNA in the supernatants were always undetectable (< $500 \mathrm{IU} / \mathrm{ml}$ for qPCR) in the gRNA-69-treated group (69-7). All viral markers in the gRNA-empty-treated group (K-15) remained at high levels. 
from the gRNA-69-treated group (subclone 69-7) was $420 \pm 278$ $\mathrm{IU} / \mathrm{ml}$. HBV cccDNA in subclone $69-7$ was undetectable by qPCR ( $<500$ copies $/ 10^{6}$ cells), whereas HBV cccDNA in subclone K15 had a concentration of $11,807,834 \pm 3,431,702$ copies $/ 10^{6}$ cells (Figures S1C,D). Additionally, the amounts of HBsAg and HBeAg in the supernatant from subclone 69-7 were always below the negative threshold $(0.05 \mathrm{IU} / \mathrm{ml}$ for HBsAg and $1 \mathrm{~S} / \mathrm{CO}$ for $\mathrm{HBeAg}$ ) for 10 consecutive days, whereas the amounts of HBsAg and $\mathrm{HBeAg}$ in the supernatant from subclone K-15 increased rapidly (Figures S1A,B). Next, to test whether this HBV clearance could be repaired, we conducted long-term observations of subclones 69-7 and K-15. In contrast to the high levels of HBeAg and HBsAg in the supernatant from subclone K-15, the amounts of $\mathrm{HBeAg}, \mathrm{HBsAg}$, and HBV DNA in the supernatant from subclone 69-7 remained below the negative critical values for 300 consecutive days; additionally, HBV cccDNA was undetectable in subclone 69-7. In contrast, all of these viral markers remained high in subclone K-15 for 300 consecutive days (Figures 3D,E).

\section{Whole Genome Sequencing of HBV-Excised Cell Line}

To assess possible off-target effects of CRISPR-Cas9 and the integrated subgenomic HBV DNA fragments in the original HBV-containing cell line (A64) and HBV-excised cell line (69-7), whole-genome sequencing (WGS) was performed. The average coverage was 71 folds in A64 and 67 folds in 69-7. In total, with human (GRCh37/hg19) genomes as reference sequences, we identified 4,981,628 SNPs, 914,579 indels and 24,344 SVs in A64 cells and 3,834,414 SNPs, 771,687 indels, and 16,719 SVs in 69-7 cells using GATK, v.2.8.1. Next, we performed an analysis of \pm 500 -bp flanking each indel against predicted gRNA69 off-target sites (Table S3). In total, we identified 6 indels in 50 potential off-target regions (Table 1), while four indels were identified in two cell lines and only two indels were different after gRNA-69 transfection, which suggests that gRNA-69 did not have significant off-target indels. Moreover, after comparing the SNPs and indels between HepG2.A64.69-7 and HepG2.A64 cells, we found that $1,175,423$ SNPs and 154,931 indels were only present in the stable HBV cell line and not the HBV-excised cell line; 28,209 SNPs and 12,039 indels were present only in the HBV-excised cell line but not in the stable HBV cell line; $3,806,205$ SNPs and 759,648 indels occurred in both samples. Thus, it means that the HBV-excised HepG2.A64.69-7 cell line missed millions of mutations compared with the stable HBV cell line HepG2.A64.

As a proof that the HBV-excised monoclone 69-7 did not exist in a very small amount as contaminants in the stable HBV cell line A64 before gRNA-69 treatment, we found a specific residual sequence of gRNA-69/Cas9 cleavage (Figure 4), which confirmed that this monoclone was generated by gRNA69/Cas9. Sanger sequencing of PCR products containing the excision site (Figure 3C) showed that after full-length HBV DNA excision by gRNA-69/Cas9 and non-homologous end joining (NHEJ) repair, three extra nucleotides "GAA" were deleted at the gRNA-69 cleavage site located in the HBV C region. Thus, we searched two 20-bp sequences (CCCGTATAAATTTGGAGCTT and CCGTATAAAGAATTTGGAGC) that could distinguish the original and excised DNA in the raw sequencing data (coverage $60 \times)$ of the two samples. The results showed that before gRNA69/Cas9 transfection, we could not find the residual sequence with the "GAA" deletion in the stable HBV cell line A64, while 11 reads containing the residual sequence with the "GAA" deletion were found in the 69-7 sequencing data. This residual sequence with the "GAA" deletion at the gRNA-69 targeting region not only indicated that the HBV-excised cell line was established by gRNA-69/Cas9, it also verified that this monoclone was not a contaminated clone in the A64 cell line.

\section{DISCUSSION}

CRISPR-Cas9 represents a potential means to radically cure chronic viral infections because this system was demonstrated to disrupt HBV cccDNA with favorable effects (Seeger and Sohn, 2014; Kennedy et al., 2015; Zhen et al., 2015a). Seeger et al. demonstrated that the use of CRISPR/Cas9 is currently the best method of functionally inactivating HBV cccDNA (Seeger and Sohn, 2016). HBV infection can induce HBV DNA fragment integration into the host genome, which had been observed in $\sim 80 \%$ of cases of HBV-induced hepatocarcinogenesis (Bréchot, 2004; Hai et al., 2014). Integrated HBV DNA could lead to aberrant regulation of host gene expression (Jiang S. et al., 2012; Jiang Z. et al., 2012; Sung et al., 2012; Xu et al., 2014) and higher genomic instability (Cha and Dematteo, 2005). Many HBV integration events occurred near or within fragile sites and other repetitive regions, such as TERT, FN1, MLL4, ROCK1, CCNE1, SENP5 (Jiang Z. et al., 2012; Sung et al., 2012; Hai et al.,

TABLE 1 | Detailed information on six called indels near gRNA-69 predicted off-target sites in A64 and 69-7 cells.








2014), Alu sequences, and microsatellites, which are prone to tumor development and progression (Feitelson and Lee, 2007). Gounder et al. demonstrated that the reduced risk of HCC was not associated with HBsAg seroclearance, and speculated that it was probably associated with the existence of HBV cccDNA and integrated HBV DNA (Gounder et al., 2016). Here, as proof of concept, we employed the CRISPR-Cas9 system to eradicate HBV infection and eliminated the persistent HBV genome, including the full-length integrated HBV DNA and HBV cccDNA, in a stable HBV cell line. These findings indicated that CRISPRCas9 could not only provide a powerful path toward a radical or "sterile" HBV cure, but also provide a means of blocking carcinogenesis by eliminating $\mathrm{HBV}$ cccDNA and integrated HBV DNA.

Using gRNAs targeting the HBV core region, the level of HBsAg and HBV DNA was reduced by an equal level as that of HBeAg (Figures 2B-D). It had been considered as a global reduction in cccDNA in this case (Seeger and Sohn, 2014; Kennedy et al., 2015; Zhen et al., 2015b) for the HBV cccDNA rapid cleavage efficiency, resulting in a high percentage of linear DNAs that is not repaired but rather destroyed (Dong et al., 2015). However, the inhibitory effect on gRNA-directly targeted genes was stronger than the effect on other HBV antigens in previous studies (Lin et al., 2014; Kennedy et al., 2015). Since the functional full-length integrated HBV DNA is also a replication root in an HBV-transgenic cell model (Sells et al., 1987) and these four effective gRNAs targeted both ends of the full-length integrated HBV DNA, we reasoned that this more thorough clearance of HBV expression was not only caused by the global reduction of cccDNA, but also by the removal of the full-length integrated HBV DNA. Another possible reason for this overall reduction was that the region we targeted, which contained the viral enhancer, X gene, and core gene(Sung et al., 2012), is essential for HBV replication or even integration (Zoulim and Locarnini, 2009). Since the specific mechanisms of HBV replication and integration remain controversial, we expect that this region will be an area of focus for future research.

In a monoclone of the stable HBV cell line 69-7, the full-length integrated $\mathrm{HBV}$ genome was excised. It is the first time, to our knowledge, that the HBV infection in a stable HBV cell line was removed thoroughly and completely using CRISPR-Cas9. At first, we aimed to value the off-target effect of gRNA-69. Unexpectedly, after sequencing the whole genome, we identified 4,981,628 SNPs in HepG2.A64 cells and only 3,834,414 SNPs were detected in the HBV-excised 69-7 cell line. We found that 1,175,423 SNPs in HepG2.A64 were absent after HBV excision. There may be two possibilities to explain this finding. Firstly, those abundant missing SNPs may be due to limited WGS coverage. Secondly, as many studies have demonstrated that HBV proteins such as HBx, HBsAg and core proteins could interfere with DNA repair [i.e., the NER (Jaitovich-Groisman et al., 2001; Lieber, 2010), BER (van de Klundert et al., 2012) or ATR (Rakotomalala et al., 2008; Wang et al., 2008)] pathways, this could have exacerbated DNA damage, by interfering with checkpoint activation, promoting cell cycle progression and ultimately leading to genetic aberrations. We speculate that those missing SNPs could have been eliminated by the re-activated DNA repair mechanism of the host. However, the specific mechanisms of $\mathrm{HBV}$ integration and $\mathrm{HBV}$ pathogenesis remain controversial, and this suggestion still needs further research.

In addition, as we established only one HBV-excised subclone without any $\mathrm{HBV}$ replication markers and missing abundant 
SNPs, there is a possibility that this HBV-excised subclone we selected is just the HepG2 cell line contaminated in HepG2.A64. To rule out this possibility, we used the WGS results with a specific residual sequence of the gRNA-69/Cas9 cleavage to evaluate the contaminant. The results showed that the HBVexcised monoclone 69-7 did not exist as contaminants in the stable HBV cell line A64 before transfection and it was generated by gRNA-69/Cas9. Unlike previous studies using CRISPR-Cas9 to disrupt HBV, we not only disrupted HBV cccDNA but also swept the full-length of integrated HBV DNA in the stable HBV cell line. In the HBV-excised cell line 69-7, HBV cccDNA, supernatant HBV DNA, HBsAg, or HBsAg could not be detected for 10 consecutive months. This showed that once the root of $\mathrm{HBV}$ replication in a stable HBV cell line had been eradicated, and HBV infection was persistently undetectable without any treatment. As the development of the next generation of sequencing technology, we could specific locate the integrated HBV DNA fragments. The developed online gRNA predicting tool could be used to design gRNAs with less off-target effect. Besides, in recent years, Adeno-Associated Virus Vectors (AAV) has captured much attention as a gene delivery system for treating human disease caused by a gene loss or mutation. The advantages of the AAV delivery scheme include its low toxicity and sustained gene expression, which can extend to 12 months after a single administration. Those properties mean that completely eradication of $\mathrm{HBV}$ infection in clinical $\mathrm{CHB}$ patients will be achieved in the near future.

\section{REFERENCES}

Bang, K. B., and Kim, H. J. (2014). Management of antiviral drug resistance in chronic hepatitis B. World J. Gastroenterol. 20, 11641-11649. doi: 10.3748/wjg. v20.i33.11641

Bloom, K., Ely, A., Mussolino, C., Cathomen, T., and Arbuthnot, P. (2013). Inactivation of hepatitis B virus replication in cultured cells and in vivo with engineered transcription activator-like effector nucleases. Mol. Ther. 21, 1889-1897. doi: $10.1038 / \mathrm{mt} .2013 .170$

Bonilla Guerrero, R., and Roberts, L. R. (2005). The role of hepatitis B virus integrations in the pathogenesis of human hepatocellular carcinoma. J. Hepatol. 42, 760-777. doi: 10.1016/j.jhep.2005.02.005

Bréchot, C. (2004). Pathogenesis of hepatitis B virus-related hepatocellular carcinoma: old and new paradigms. Gastroenterology 127(5 Suppl. 1), S56-S61. doi: 10.1053/j.gastro.2004.09.016

Cha, C., and Dematteo, R. P. (2005). Molecular mechanisms in hepatocellular carcinoma development. Best Pract. Res. Clin. Gastroenterol. 19, 25-37. doi: 10.1016/j.bpg.2004.11.005

Chen, J., Zhang, W., Lin, J., Wang, F., Wu, M., Chen, C., et al. (2014). An efficient antiviral strategy for targeting hepatitis B virus genome using transcription activator-like effector nucleases. Mol. Ther. 22, 303-311. doi: $10.1038 / \mathrm{mt} .2013 .212$

Cradick, T. J., Keck, K., Bradshaw, S., Jamieson, A. C., and McCaffrey, A. P. (2010). Zinc-finger nucleases as a novel therapeutic strategy for targeting hepatitis B virus DNAs. Mol. Ther. 18, 947-954. doi: 10.1038/mt.2010.20

Dandri, M., Burda, M. R., Will, H., and Petersen, J. (2000). Increased hepatocyte turnover and inhibition of woodchuck hepatitis B virus replication by adefovir in vitro do not lead to reduction of the closed circular DNA. Hepatology 32, 139-146. doi: 10.1053/jhep.2000.8701

Dong, C., Qu, L., Wang, H., Wei, L., Dong, Y., and Xiong, S. (2015). Targeting hepatitis B virus cccDNA by CRISPR/Cas9 nuclease efficiently inhibits viral replication. Antiviral Res. 118, 110-117. doi: 10.1016/j.antiviral.2015.03.015

\section{AUTHOR CONTRIBUTIONS}

HL, CS, YS, HS, and SQ conceived the study. HL, SW, and CS cultured cells and isolated genomic DNA. CS, HL, YH, HBL, YT, and LY processed experiments and analyzed the WGS data. QL, PL, YL, ML, JZ, CY, XY, LJ, JX, LW, RH, and DX shared regents and materials. All authors read and approved the final manuscript.

\section{FUNDING}

This work was supported by grants from the National Nature Science Foundation of China (81371854), the Beijing Nature Science Foundation (7162145), and the Innovation Foundation of AMMS (2015CXJJ26).

\section{ACKNOWLEDGMENTS}

We thank Junfeng Li, Dayang Zou, Zhihui Xu, and Qi Li for discussions and reagents. We also thank the Research Center for Liver Failure, Beijing 302nd Hospital for technical support.

\section{SUPPLEMENTARY MATERIAL}

The Supplementary Material for this article can be found online at: http://journal.frontiersin.org/article/10.3389/fcimb. 2017.00091/full\#supplementary-material

Feitelson, M. A., and Lee, J. (2007). Hepatitis B virus integration, fragile sites, and hepatocarcinogenesis. Cancer Lett. 252, 157-170. doi: 10.1016/j.canlet. 2006.11.010

Gish, R. G., Given, B. D., Lai, C. L., Locarnini, S. A., Lau, J. Y., Lewis, D. L., et al. (2015). Chronic hepatitis B: virology, natural history, current management and a glimpse at future opportunities. Antiviral Res. 121, 47-58. doi: 10.1016/j.antiviral.2015.06.008

Gounder, P. P., Bulkow, L. R., Snowball, M., Negus, S., Spradling, P. R., Simons, B. C., et al. (2016). Nested case-control study: hepatocellular carcinoma risk after hepatitis B surface antigen seroclearance. Aliment. Pharmacol. Ther. 43, 1197-1207. doi: 10.1111/apt.13621

Guo, J. T., and Guo, H. (2015). Metabolism and function of hepatitis B virus cccDNA: implications for the development of cccDNA-targeting antiviral therapeutics. Antiviral Res. 122, 91-100. doi: 10.1016/j.antiviral.2015.08.005

Hai, H., Tamori, A., and Kawada, N. (2014). Role of hepatitis B virus DNA integration in human hepatocarcinogenesis. World J. Gastroenterol. 20, 6236-6243. doi: 10.3748/wjg.v20.i20.6236

Hsu, P. D., Scott, D. A., Weinstein, J. A., Ran, F. A., Konermann, S., Agarwala, V., et al. (2013). DNA targeting specificity of RNA-guided Cas9 nucleases. Nat. Biotechnol. 31, 827-832. doi: 10.1038/nbt.2647

Hu, W., Kaminski, R., Yang, F., Zhang, Y., Cosentino, L., Li, F., et al. (2014). RNA-directed gene editing specifically eradicates latent and prevents new HIV-1 infection. Proc. Natl. Acad. Sci. U.S.A. 111, 11461-11466. doi: 10.1073/pnas.1405186111

Jaitovich-Groisman, I., Benlimame, N., Slagle, B. L., Perez, M. H., Alpert, L., Song, D. J., et al. (2001). Transcriptional regulation of the TFIIH transcription repair components XPB and XPD by the hepatitis B virus $\mathrm{x}$ protein in liver cells and transgenic liver tissue. J. Biol. Chem. 276, 14124-14132. doi: 10.1074/jbc.M010852200

Jiang, S., Yang, Z., Li, W., Li, X., Wang, Y., Zhang, J., et al. (2012). Reevaluation of the carcinogenic significance of hepatitis $B$ virus integration in hepatocarcinogenesis. PLoS ONE 7:e40363. doi: 10.1371/journal.pone.0040363 
Jiang, Z., Jhunjhunwala, S., Liu, J., Haverty, P. M., Kennemer, M. I., Guan, Y., et al. (2012). The effects of hepatitis B virus integration into the genomes of hepatocellular carcinoma patients. Genome Res. 22, 593-601. doi: $10.1101 /$ gr.133926.111

Kao, J. H., and Chen, D. S. (2002). Global control of hepatitis B virus infection. Lancet Infect. Dis. 2, 395-403. doi: 10.1016/S1473-3099(02)00315-8

Karimova, M., Beschorner, N., Dammermann, W., Chemnitz, J., Indenbirken, D., Bockmann, J. H., et al. (2015). CRISPR/Cas9 nickase-mediated disruption of hepatitis B virus open reading frame S and X. Sci. Rep. 5:13734. doi: $10.1038 /$ srep 13734

Kennedy, E. M., Bassit, L. C., Mueller, H., Kornepati, A. V., Bogerd, H. P., Nie, T., et al. (2015). Suppression of hepatitis B virus DNA accumulation in chronically infected cells using a bacterial CRISPR/Cas RNA-guided DNA endonuclease. Virology 476, 196-205. doi: 10.1016/j.virol.2014.12.001

Li, H., Sheng, C., Liu, H., Liu, G., Du, X., Du, J., et al. (2016). An Effective molecular target site in hepatitis $\mathrm{B}$ virus $\mathrm{S}$ gene for Cas9 cleavage and mutational inactivation. Int. J. Biol. Sci. 12, 1104-1113. doi: 10.7150/ijbs.16064

Lieber, M. R. (2010). The mechanism of double-strand DNA break repair by the nonhomologous DNA end-joining pathway. Annu. Rev. Biochem. 79, 181-211. doi: 10.1146/annurev.biochem.052308.093131

Lin, S. R., Yang, H. C., Kuo, Y. T., Liu, C. J., Yang, T. Y., Sung, K. C., et al. (2014). The CRISPR/Cas9 system facilitates clearance of the intrahepatic hbv templates in vivo. Mol. Ther. Nucleic Acids 3:e186. doi: 10.1038/mtna.2014.38

Liu, W., Song, H., Chen, Q., Xu, C., Zhang, W., Liu, Y., et al. (2016). Multidrug resistance protein 4 is a critical protein associated with the antiviral efficacy of nucleos(t)ide analogues. Liver Int. 36, 1284-1294. doi: 10.1111/liv.13104

Liu, X., Hao, R., Chen, S., Guo, D., and Chen, Y. (2015). Inhibition of hepatitis $\mathrm{B}$ virus by CRISPR/Cas9 system via targeting the conserved regions of viral genome. J. Gen. Virol. doi: 10.1099/vir.0.000159

Mali, P., Yang, L., Esvelt, K. M., Aach, J., Guell, M., DiCarlo, J. E., et al. (2013). RNA-guided human genome engineering via Cas9. Science 339, 823-826. doi: $10.1126 /$ science. 1232033

Matsubara, K., and Tokino, T. (1990). Integration of hepatitis B virus DNA and its implications for hepatocarcinogenesis. Mol. Biol. Med. 7, 243-260.

Moraleda, G., Saputelli, J., Aldrich, C. E., Averett, D., Condreay, L., and Mason, W. S. (1997). Lack of effect of antiviral therapy in nondividing hepatocyte cultures on the closed circular DNA of woodchuck hepatitis virus. J. Virol. 71, 9392-9399.

Nassal, M. (2015). HBV cccDNA: viral persistence reservoir and key obstacle for a cure of chronic hepatitis B. Gut 64, 1972-1984. doi: 10.1136/gutjnl-2015309809

Peng, C., Lu, M., and Yang, D. (2015). CRISPR/Cas9-based tools for targeted genome editing and replication control of HBV. Virol. Sin. 30, 317-325. doi: $10.1007 / \mathrm{s} 12250-015-3660-\mathrm{x}$

Qi, L. S., Larson, M. H., Gilbert, L. A., Doudna, J. A., Weissman, J. S., Arkin, A. P., et al. (2013). Repurposing CRISPR as an RNA-guided platform for sequence-specific control of gene expression. Cell 152, 1173-1183. doi: 10.1016/j.cell.2013.02.022

Rakotomalala, L., Studach, L., Wang, W. H., Gregori, G., Hullinger, R. L., and Andrisani, O. (2008). Hepatitis B virus X protein increases the Cdt1-togeminin ratio inducing DNA re-replication and polyploidy. J. Biol. Chem. 283, 28729-28740. doi: 10.1074/jbc.M802751200

Ramanan, V., Shlomai, A., Cox, D. B., Schwartz, R. E., Michailidis, E., Bhatta, A., et al. (2015). CRISPR/Cas9 cleavage of viral DNA efficiently suppresses hepatitis B virus. Sci. Rep. 5:10833. doi: 10.1038/srep10833

Ran, F. A., Hsu, P. D., Wright, J., Agarwala, V., Scott, D. A., and Zhang, F. (2013). Genome engineering using the CRISPR-Cas9 system. Nat. Protoc. 8, 2281-2308. doi: 10.1038/nprot.2013.143

Roberts, L. R., and Gores, G. J. (2005). Hepatocellular carcinoma: molecular pathways and new therapeutic targets. Semin. Liver Dis. 25, 212-225. doi: $10.1055 / \mathrm{s}-2005-871200$

Seeger, C., and Sohn, J. A. (2014). Targeting hepatitis B virus with CRISPR/Cas9. Mol. Ther. Nucleic Acids 3:e216. doi: 10.1038/mtna.2014.68

Seeger, C., and Sohn, J. A. (2016). Complete spectrum of CRISPR/Cas9-induced mutations on HBV cccDNA. Mol. Ther. 24, 1258-1266. doi: 10.1038/mt. 2016.94

Sells, M. A., Chen, M. L., and Acs, G. (1987). Production of hepatitis B virus particles in Hep G2 cells transfected with cloned hepatitis B virus DNA. Proc. Natl. Acad. Sci. U.S.A. 84, 1005-1009. doi: 10.1073/pnas.84.4.1005
Seo, Y., and Yano, Y. (2014). Short- and long-term outcome of interferon therapy for chronic hepatitis B infection. World J. Gastroenterol. 20, 13284-13292. doi: 10.3748/wjg.v20.i37.13284

Sharon, A., and Chu, C. K. (2008). Understanding the molecular basis of HBV drug resistance by molecular modeling. Antiviral Res. 80, 339-353. doi: 10.1016/j.antiviral.2008.07.010

Shaw, T., Bartholomeusz, A., and Locarnini, S. (2006). HBV drug resistance: mechanisms, detection and interpretation. J. Hepatol. 44, 593-606. doi: 10.1016/j.jhep.2006.01.001

Sung, W. K., Zheng, H., Li, S., Chen, R., Liu, X., Li, Y., et al. (2012). Genome-wide survey of recurrent $\mathrm{HBV}$ integration in hepatocellular carcinoma. Nat. Genet. 44, 765-769. doi: 10.1038/ng.2295

Tarocchi, M., Polvani, S., Marroncini, G., and Galli, A. (2014). Molecular mechanism of hepatitis B virus-induced hepatocarcinogenesis. World J. Gastroenterol. 20, 11630-11640. doi: 10.3748/wjg.v20.i33.11630

Tuttleman, J. S., Pourcel, C., and Summers, J. (1986). Formation of the pool of covalently closed circular viral DNA in hepadnavirus-infected cells. Cell 47, 451-460. doi: 10.1016/0092-8674(86)90602-1

van de Klundert, M. A., van Hemert, F. J., Zaaijer, H. L., and Kootstra, N. A. (2012). The hepatitis B virus $\mathrm{x}$ protein inhibits thymine DNA glycosylase initiated base excision repair. PLoS ONE 7:e48940. doi: 10.1371/journal.pone.0048940

Wang, J., Xu, Z. W., Liu, S., Zhang, R. Y., Ding, S. L., Xie, X. M., et al. (2015). Dual gRNAs guided CRISPR/Cas9 system inhibits hepatitis B virus replication. World J. Gastroenterol. 21, 9554-9565. doi: 10.3748/wjg.v21.i32.9554

Wang, W. H., Hullinger, R. L., and Andrisani, O. M. (2008). Hepatitis B virus $\mathrm{X}$ protein via the p38MAPK pathway induces E2F1 release and ATR kinase activation mediating p53 apoptosis. J. Biol. Chem. 283, 25455-25467. doi: 10.1074/jbc.M801934200

Wei-ming, Y., Yan, L., Lan-lan, S., Zhi-hui, X., Peng-gao, L., Lv-ping, B., et al. (2014). Inhibitory effect of herbal concoction Suduxing on hepatitis B virus replication in vitro (in Chinese). Med. J. Chin. PLA 39:4. doi: 10.11855/j.issn.0577-7402.2014.02.08

Xu, C., Zhou, W., Wang, Y., and Qiao, L. (2014). Hepatitis B virus-induced hepatocellular carcinoma. Cancer Lett. 345, 216-222. doi: 10.1016/j.canlet.2013. 08.035

Zhang, F., Wen, Y., and Guo, X. (2014). CRISPR/Cas9 for genome editing: progress, implications and challenges. Hum. Mol. Genet. 23, R40-R46. doi: $10.1093 / \mathrm{hmg} / \mathrm{ddu} 125$

Zhen, S., Hua, L., Liu, Y. H., Gao, L. C., Fu, J., Wan, D. Y., et al. (2015a). Harnessing the clustered regularly interspaced short palindromic repeat (CRISPR)/CRISPR-associated Cas9 system to disrupt the hepatitis B virus. Gene Ther. 22, 404-412. doi: 10.1038/gt.2015.2

Zhen, S., Hua, L., Liu, Y. H., Gao, L. C., Fu, J., Wan, D. Y., et al. (2015b) Harnessing the clustered regularly interspaced short palindromic repeat (CRISPR)/CRISPR-associated Cas9 system to disrupt the hepatitis B virus. Gene Ther. 22, 404-412. doi: 10.1038/gt.2015.2

Zhong, Y., Han, J., Zou, Z., Liu, S., Tang, B., Ren, X., et al. (2011). Quantitation of HBV covalently closed circular DNA in micro formalin fixed paraffinembedded liver tissue using rolling circle amplification in combination with real-time PCR. Clin. Chim. Acta 412, 1905-1911. doi: 10.1016/j.cca.2011. 06.031

Zoulim, F., and Locarnini, S. (2009). Hepatitis B virus resistance to nucleos(t)ide analogues. Gastroenterology 137, 1593-1608.e2. doi: 10.1053/j.gastro.2009. 08.063

Zucman-Rossi, J., and Laurent-Puig, P. (2007). Genetic diversity of hepatocellular carcinomas and its potential impact on targeted therapies. Pharmacogenomics 8, 997-1003. doi: 10.2217/14622416.8.8.997

Conflict of Interest Statement: The authors declare that the research was conducted in the absence of any commercial or financial relationships that could be construed as a potential conflict of interest.

Copyright (c) 2017 Li, Sheng, Wang, Yang, Liang, Huang, Liu, Li, Yang, Yang, Jia, Xie, Wang, Hao, Du, Xu, Zhou, Li, Sun, Tong, Li, Qiu and Song. This is an open-access article distributed under the terms of the Creative Commons Attribution License (CC $B Y)$. The use, distribution or reproduction in other forums is permitted, provided the original author(s) or licensor are credited and that the original publication in this journal is cited, in accordance with accepted academic practice. No use, distribution or reproduction is permitted which does not comply with these terms. 